\title{
EFECTOS DEL VACIADO DEL EMBALSE DE BARASONA EN LAS COMUNIDADES DE PECES FLUVIALES
}

\author{
M. Morillo y D. García de Jalón \\ Laboratorio de Hidrobiología.E.T.S.I. de Montes, Universidad Politécnica de Madrid. Avda. Ramiro de Maeztu s/n, \\ 28040 Madrid.
}

\section{RESUMEN}

Con objeto de evaluar los efectos del vaciado del Embalse de Barasona en las comunidades piscícolas fluviales se realizaron muestreoa cuantitativos en tramos situados aguas arriba del embalse (río Jsábena) y aguas abajo (ríos Ésera y Cinca). Se realizaron 5 muestreos antes del primer desembalse, y 5 posteriores.

El vaciado ha afectado de manera mas drástica, paradójicamente, a las coniunidades de aguas arriba del embalse del río Isábena, que han visto reducidas sus biomasas como sus densidades. Esto parece perfectamente lógico dado que el embalse actuaba a modo de reservorio alojando a los individuos de mayor tamaño, reproductores potenciales.

En los tramos de aguas abajo el desembalsado ejerció un efecto de "barrido" de todo lo que hubiera aguas abajo (preferentemente individuos grandes que no pueden encontrar refugio ante la crecida de las aguas). Pero en el Ésera, dado que las poblaciones eran escasas y estaban inal estructuradas (debido a la intensa regulación de caudales con tina autencia total de caudales ecológicos a lo largo muchos años) apenas se observan diferencias, tan solo un ligero incremento en el tamaño de las mismas. En el río Cinca los efectos tampoco fueron patentes, debido a que está más alejado de la presa y tener un cauce mayor.

Palabras Clave: desembalse, impacto, peces fluviales.

\section{ABSTRACT}

A sampling program was undertaken in order to evaluate the effects of complete empty out of Barasona Reservoir on the downstream fish communities. Five surveys before first depletion and 5 after it were performed. Three stream reaches were sampled one downstream the reservoir (Isabena river) and other two below (Esera river and Cinca river).

Fisheries upstream the reservoir were badly affected by the empty out, leading to by significant reductions on their densities and biomass. These fisheries, were composed by barbel and nase, use Isabena river as spawning and rearing habitat, while adults living in the reservoir where flush out of it. In the downstream reaches the effect of the reservoir emptying was less marked because fisheries were already disturbed by the extremety low flow regulation and new continuos water releases which causes upstream migrations to compensate first flushing effects.

Key Words: reservoir flushing, impacts, stream fishes

\section{INTRODUCCIÓN}

Las cuencas hidrográficas con problemas intensos de erosión en sus laderas, afectan a los ríos que de ellas drenan, produciendo un gran aporte de sedimentos finos. Estos finos perturban grandemente estos ecosistemas fluviales, principalmente por colmatación del medio intersticial de sus fondos, que es el factor limitante de la capacidad biogénica de la mayoría de los ríos. Si en este tipo de ríos con gran cantidad de finos se construye una presa, su embalse se convierte en una gigantesca trampa de sedimentos, y los consiguientes riesgos de colmatación del vaso son muy altos. Este es el caso del embalse de Baraso- na en el río Ésera, cuya correcta gestión exige un vaciado periódico.

En el presente trabajo se han evaluado los efectos que ha presentado el vaciado del embalse de Barasona en la ictiofauna de varios ríos de la cuenca del Ésera y del Cinca en la provincia de Huesca. Esta labor queda incluida dentro de un proyecto más amplio cuya finalidad Última era concluir en un conocimiento profundo del estado actual del ecosistema que se ordena en torno al embalse y determinar la incidencia que desencadenaría el vaciado de dicha obra, estableciendo un plan conjunto de actuaciones.

El vaciado de embalses es una actividad de la gestión de los embalses que debe realizarse perió- 
dicamente con objeto de asegurar el correcto funcionamiento de la presa y compensar en lo posible el aterramiento de su vaso. Sin embargo, existen pocos trabajos que nos evalúan los efectos de estos desembalses en la comunidades ícticas. Entre ellos debe ser citado el de Granado (1981).

\section{METODOLOGíA}

Para la realización del estudio se realizaron once campañas de muestreo, cinco antes del vaciado (correspondientes a los meses de febrero, julio, septiembre y diciembre de 1994 y mayo de 1995) y seis después (correspondientes a los meses de julio, septiembre y noviembre de 1996, y junio y septiembre de 1997).

Como método de captura se seleccionó la pesca eléctrica. El método de muestreo seleccionado fue el de extracción sin reemplazo, por su sencillez y el menor trabajo y tiempo que conllevan sus operaciones. Se dieron un mínimo de tres pasadas en cada punto, con una cuarta adicional si las sucesivas capturas seguían una pauta poco adecuada.

Los peces capturados fueron estabulados en barreños con agua fresca y tranquilizante MS222. De cada ejemplar se anotaron longitud hasta la horquilladura, peso y, en los casos que fuera posible, el sexo. Para la determinación de la edad se tomaron escamas de una submuestra de individuos capturados (seleccionando la zona entre la aleta dorsal y la línea lateral). La determinación de la edad de los ejemplares se realizó mediante la lectura de las mismas, con ayuda de los gráficos de frecuencia de longitudes o gráficos de Petersen (en García de Jalón et al, 1993).

Para la estimación del número total de individuos capturados en cada muestra se utilizó el método de Máxima Verosimilitud Ponderada (Carle \& Strub, 1978) por su mayor robustez estadística (Cowx, 1983).

El tratamiento estadístico de los datos se realizó por medio del paquete estadístico Statgraphics Plus V 4.0 para Windows.

\section{ÁREA DE ESTUDIO}

Los tramos de río estudiados se encuentran en las inmediaciones del embalse de Barasona, a la altura de los términos municipales de Graus y Puebla de Castro, en la provincia de Huesca. El estudio de las poblaciones ícticas se realizó en cuatro puntos de muestreo situados en el río Ésera (estaciones ES-1 y ES-3), Isábena (estación ISA-1), y Cinca (estación CI-2) (Fig. 1). A continuación se hace una descripción más detallada de los puntos de muestreo:

- Río Ésera:

Estación ES-1: localizada en el punto 31TBG818812 en coordenadas U.T.M., a la altura del km. 43.3 de la carretera comarcal 139, aguas arriba del embalse de Barasona o Joaquín Costa. El río atraviesa una zona relativamente abierta, con presencia de cultivos en los márgenes.

Estación ES-3: situada aguas abajo del cruce entre la carretera comarcal 1311 y la carretera local a Olvena, antes de la confluencia del río Ésera con el Cinca, en el punto 31TBG723650.

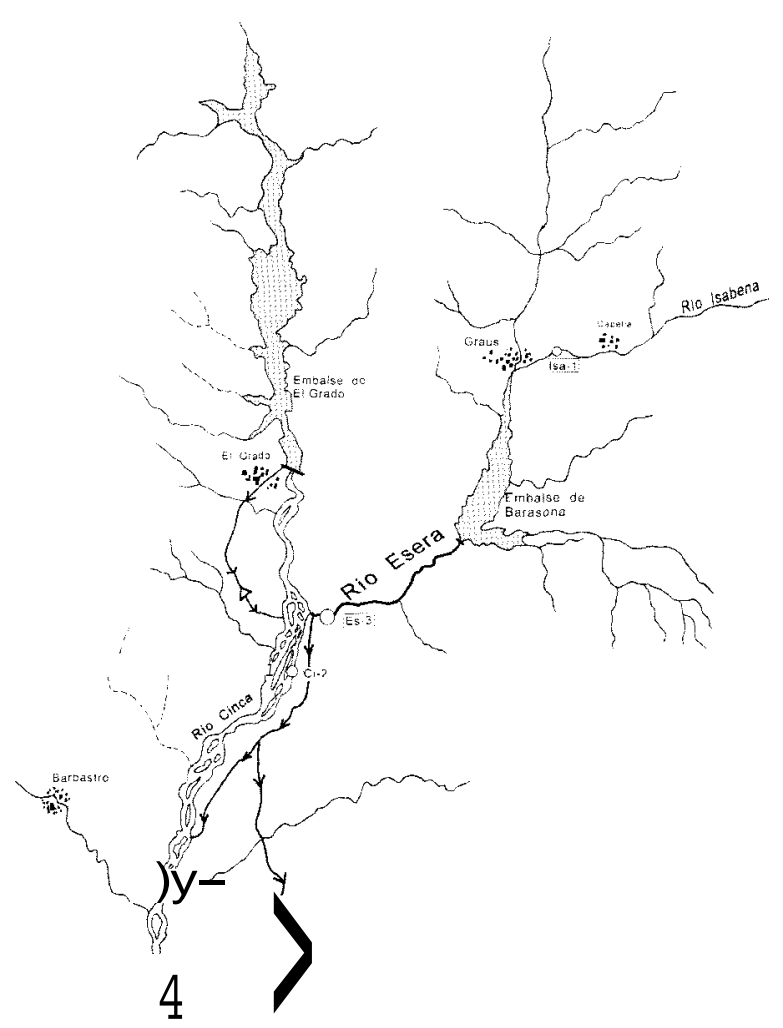

Figura 1. Localización de las estaciones de muestreo. Location of sampling stations. 
- Río Isábena:

Estación ISA-1: localizada en el punto 31TBG830742, en las proximidades del km 3 de la carretera que une las localidades de Graus y Capella. En este punto el río está dividido en dos brazos, los cuales se muestrearon sistemáticamente.

\section{- Río Cinca:}

Estación CI-2: con coordenadas U.T.M. 31 TBG7 13628, situada junto al km 30.6 de la carretera comarcal 1311, aguas abajo del azud Arias 1.

\section{RESULTADOS}

El primer desembalse tuvo lugar a principios de Octubre del 95 y arrastró sedimentos del vaso del embalse que fueron depositándose en el lecho de los tramos aguas abajo. Posteriormente, las fuertes precipitaciones del otoño del 9.5 llenaron el embalse y se realizaron sueltas de fuertes caudales que limpiaron de finos los tramos fluviales estudiados (situación detectada en el muestreo de Julio-96). Durante el verano, se soltó o se filtró aguas de fondo del embalse, que arrastraban sedimentos del mismo. Como el caudal era pequeño (aunque mantenido), estos finos se sedimentaron inmediatamente aguas abajo del embalse. Según avanzaba el verano la concentración de finos aumentó, consecuencia de irse vaciando el embalse, y el tramo de río Esera cubierto de sedimentos alcanzó a su desembocadura con el Cinca. Y finalmente las lluvias del otoño del 96 volvieron a permitir crecidas que eliminaron los sedimentos del lecho fluvial, condiciones que se mantuvieron a lo largo del invierno y primavera del 97. Durante el verano (Junio 97) debido al elevado caudal circulante las aguas permanecieron transparentes y los fondos limpios de finos. No obstante, en septiembre con el descenso del caudal, vuelve a aparecer una turbidez que impide ver el lecho del río.

Los muestreos de la estación del río Ésera (ES3) detectaron estos cambios: Julio con substrato de cantos rodados y aguas limpias; Septiembre con una capa de sedimentos finos de más 2.5 centímetros y aguas turbias; y Noviembre vuelta a las condiciones anteriores. Las comunidades piscícolas reaccionaron al impacto del desembalse y también a estas fluctuaciones posteriores, por lo que los efectos del primero quedan parcialmente enmascarados.

Además, debemos tener en cuenta que las comunidades existentes previas al desembalse vivían en un régimen de caudales que era prácticamente nulo durante todo el año, (limitado a las filtraciones de la presa) salvo cuando excepcionalmente las avenidas del río se encontraban el embalse casi lleno y se vertía por coronación de presa con caudales que superaban $\operatorname{los} 100 \mathrm{~m}^{3} / \mathrm{s}$. Después del vaciado este régimen cambia y se instaura un caudal base que puede haber hecho las veces de un caudal ecológico mínimo. Dicho cambio de régimen de caudales probablemente ha compensado de alguna manera las condiciones adversas provocadas por los arrastres del vaciado y por sedimentación de finos.

\section{Las comunidades ícticas}

En los tramos muestreados de los ríos Isábena, Ésera y Cinca se han encontrado las siguientes especies: trucha común (Salmo trutta, L.), carpa común y real (Cyprinus carpio, L.), madrilla (Chondrostoma polylepis, L.), barbo de Graells (Barbus graellsii, Steindachner) y culirroyo (Barbus haasi, Mertens), bagre (Leuciscus cephalus, L.), gobio (Gobio gobio, L.), pez lobo (Noemaechiulus barbatulus (L.)), black-bass (Micropterus salmoides (Lacépède)) y blenio de río (Blennius fluviatilis, Asso).

De estas especies, la madrilla y el barbo son las únicas que aparecen en todas las estaciones muestreadas. No existe ninguna especie que se localice sólo en los tramos por encima del embalse mientras que hay seis que tan sólo se encuentran en los ríos aguas abajo de la presa: carpa, bagre, gobio, pez lobo, black-bass y bienio. La carpa y el blackbass son especies de aguas leníticas que han sido introducidas en el embalse y que desaparecen tras el vaciado (tan solo se capturó un ejemplar de carpa en el río Ésera debajo de la presa (ES-3), donde puede habitar debido al escaso caudal que suelta la presa. Por el contrario, el blenio y el pez lobo son especies autóctonas de tramos más altos cuyo ascenso se ve limitado por la presa.

\section{Poblaciones ícticas aguas arriba del embalse}

En los ríos aguas arriba del embalse de Barasoria hemos encontrado poblaciones de madrilla, barbo común y culirroyo y trucha co- 
Tabla 1. Densidades y biomasas niedias (desviaciones estándar entre paréntesis) de las poblaciones piscícolas del río Isábena antes y después del desembalse. (* se observan diferencias significativas para $\mathrm{p}<0.05$ ). River Isábena fish population density and biomass (with standard deviation). Comparison between before and after the withdrawal (* significative differences $p<0.05$ ).

\begin{tabular}{lcccc}
\hline & & río Isábena (isa-1) & densidad (ind/m2) & biomasa (gr/m2) \\
\hline & antes & después & antes & después \\
\hline Ch. toxostoma & $0.115(0.090)$ & $0.258(0.345)$ & $0.58(0.10)$ & $0.87(1.35)$ \\
B. graellsii & $0.645(0.847)$ & $0.246(0.376)$ & $17.99(9.38)$ & $2.35(3.35)^{*}$ \\
TOTAL & $0.760(0.896)$ & $0.553(0.479)$ & $17.49(9.57)$ & $8.56(12.63)$ \\
\hline
\end{tabular}

mún. En el caso de los barbos, dado que no se pudieron diferenciar las dos especies en los individuos jóvenes, se han considerado como una sola especie en el tratamiento de los datos.

La distribución de estas especies se refleja en sus respectivas abundancias por estación, presentadas en forma de densidad y biomasa. Estos parámetros se han calculado para las poblaciones que aparecieron en suficiente cantidad en cada estación. Los datos concretos se indican en la Tabla 1.

\section{Río Ésera}

En el río Ésera tan solo se pudieron realizar muestreos de tipo cuantitativo antes del vaciado. Después, los elevados caudales circulantes impidieron el cierre del tramo con lo cual tan solo se realizaron muestreos de tipo cualitativo. Pese a ello, podemos observar que la comunidad íctica está dominada por la madrilla, seguida del barbo común como especie subdominante, y con el barbo culirroyo y la trucha común como especies acompañantes.

\section{Río Isábena}

Las condiciones naturales del Isábena permiten un buen hábitat capaz de albergar abundantes poblaciones de peces. La comunidad de este río está dominada por el barbo antes y después del desembalse, con la excepción del muestreo de septiembre de 1996, en el que es sustituido por la madrilla (Fig.2). El río Isábena es, por tanto, muchísimo más "barbero" que el Ésera. Las razones por las que los barbos prefieran el Isábena, que es un río mas pequeño que el Esera y por lo tanto con menos capacidad para ofrecer refugio, pueden estar en el hecho de que el río Ésera, en el tramo muestreado, está sometido a unas fuertes y repentinas fluctuaciones de caudal (causadas por saltos hidroeléctricos localizados aguas arriba esta estación).

El efecto del desembalse también lo detectan las comunidades de aguas arriba del embalse, debido a que estos tramos son las zonas de freza de las especies reófilas que habitan el embalse. De este modo la densidad de la comunidad íctica se redujo a poco más de la mitad y sobre todo su biomasa disminuyó de unos valores medios de $18.58 \mathrm{~g} / \mathrm{m}^{2}$ a $1.09 \mathrm{~g} / \mathrm{m}^{2}$ después del desembalse, lo que supone una reducción aproximada del $94 \%$. Esta disminución, si bien carece de significación estadística $(p<0.05$ debido probablemente a la fluctuabilidad de los datos), tiene un significado biológico evidente: al desaparecer el embalse, la mayor parte de los adultos de barbo y madrilla que en él se alojaban son arrastrados durante el vaciado, dejando de subir a desovar al Isábena y, por consiguiente disminuye la biomasa íctica.

Por especies, la población de barbo sufre fluctuaciones estacionales debidas a las migraciones reproductivas (similares a las detectadas en el río Ésera) que siguen patrones distintos antes y después del desembalse. Antes del vaciado, la población alcanza su máxima densidad en el mes de septiembre, coincidiendo con un elevado porcentaje de alevines. Después del vaciado, en cambio, la máxima densidad se alcanza en junio, mes en el que la población está compuesta en su mayor parte por alevines $(89 \%$ del total). Con la biomasa se observa un cambio similar. Así antes del desembalse, la biomasa máxima se alcanza en julio, debido a la presencia de grandes reproductores que suben para frezar. Después del vaciado, en 

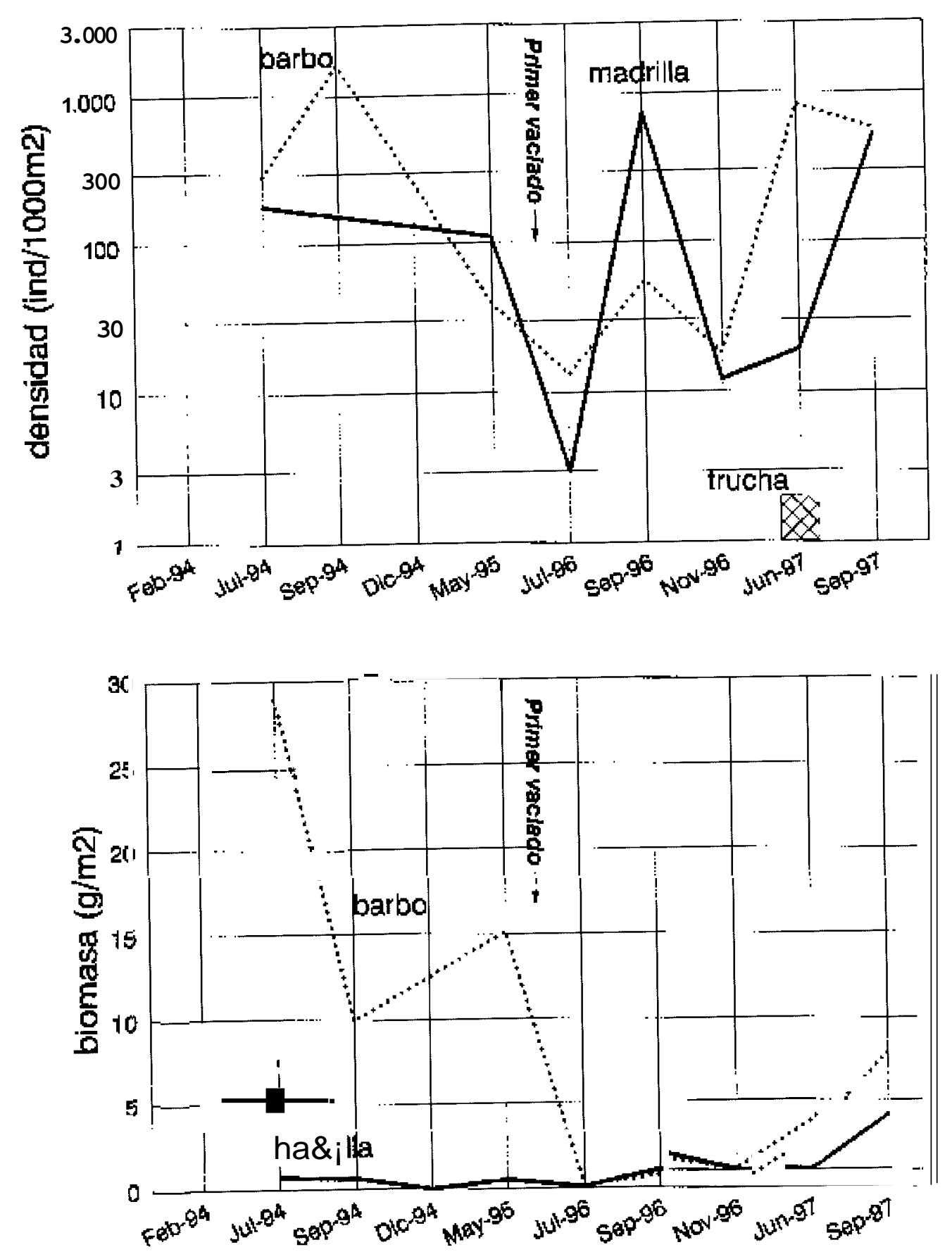

Figura 2. Evolución de la densidad y la biomasa de las distintas poblaciones ícticas del río Isábena (ISA-1) a lo largo del estudio. Density and biomass evolution of fish populations at Isabena river (ISA-1) during the study period. 
cambio, los valores máximos de biomasa se alcanzan en el mes de junio $\left(0.82 \mathrm{ind} / \mathrm{m}^{2}\right)$, coincidiendo también con un elevado porcentaje de alevines $(68 \%)$. Esto es debido a la desaparición de todos los individuos grandes ( $>3+$ ) durante el desembalse, que genera un descenso estadísticamente significativo de la biomasa de barbo (Tabla 1).

Con la madrilla se observa un efecto similar aunque menos marcado en la biomasa, cuyos valores medios aumentan de $0.58 \mathrm{~g} / \mathrm{m}^{2}$ antes del vaciado a $0.87 \mathrm{~g} / \mathrm{m}^{2}$ después, no observándose diferencias significativas entre ambos periodos (Tabla 1). La densidad también aumenta, duplicando su valor después del vaciado $\left(0.11 \mathrm{ind} / \mathrm{m}^{2}\right.$ a 0.26 ind $/ \mathrm{m}^{2}$ ) (Fig. 2).

Por Último hay que mencionar la misteriosa aparición de una carpa de pequeño tamaño, que probablemente proceda de las repoblaciones efectuadas en el embalse después del vaciado.

\section{Poblaciones ícticas aguas abajo del embalse}

En los tramos muestreados aguas abajo del embalse hemos encontrado poblaciones de madrilla, barbo y gobio acompañados de trucha, blenio, pez lobo, bagre, carpa y black-bass.

\section{Río Ésera}

Los resultados, de los muestreos cuantitativos en el tramo del Esera aguas abajo de la presa se exponen en la Tabla 2, valores, todos ellos muy bajos en términos globales. No obstante se puede observar un fuerte aumento de la comunidad íctica en términos de densidad, cuyos valores medios pasan de $0.07 \mathrm{ind} / \mathrm{m}^{2}$ a $0.22 \mathrm{ind} / \mathrm{m}^{2}$, y biomasa, con valores de $0.85 \mathrm{~g} / \mathrm{m}^{2}$ antes del vaciado y de $1.05 \mathrm{~g} / \mathrm{m}^{2}$ después (Tabla 2).

Por especies se observa que la madrilla prácticamente desaparece después del primer vaciado, recolonizando este tramo al año siguiente por alevines. No obstante, sigue siendo la especie dominante (o codominante) de la comunidad. Su población sigue creciendo y en septiembre de 1997 alcanza la mayor densidad estimada a lo largo de todo el estudio, $0.11 \mathrm{ind} / \mathrm{m}^{2}$ (Tabla 2). Sin embargo, su biomasa después del desembalse es bastante baja, no recuperando en ningún momento los valores estimados antes del mismo (Fig.3). Esto se debe al rejuvenecimiento de la población. En efecto, el análisis de su estructura poblacional muestra que antes del desembalsado presenta una estructura relativamente estable, con seis clases de edad $(0+$ a $5+)$, de las cuales $0+$ y $3+$ eran las más representadas $(30 \%$ y $23 \%$ respectivamente). Sin embargo, después del vaciado la mayoría de los adultos han desaparecido, probablemente arrastrados por las aguas, lo que explica el aumento de densidad de la comunidad observado, sin el correspondiente aumento de biomasa.

El gobio tan solo aparece en los tramos situados aguas abajo del embalse y sus poblaciones nunca son abundantes (densidad media $=0.025$ $\mathrm{ind} / \mathrm{m}^{2}$ y $0.08 \mathrm{ind} / \mathrm{m}^{2}$ antes y después del vaciado). Sin embargo, en otoño (sep/dic-94) es la especie dominante de la comunidad. Este hecho probablemente se debe al uso de este tramo del río como zona de alevinaje. Después del primer vaciado (año 1996). la población se reduce drásticamente, probablemente a causa de cambios en el hábitat (desaparición de macrófitas cubiertas de sedimentos) volviéndose a recuperar a lo largo de 1997.

En cuanto a su estructura poblacional se refiere, ésta se mantiene relativamente estable, con tres clases de edad $0+$ a $2+$ siendo los alevines el grupo dominante.

Tabla 2. Densidad y biomasas medias (desviaciones estándar entre paréntesis) de las poblaciones ícticas del río Esera aguas abajo del embalse de Barasona antes y después del desembalse. * se observan diferencias significativas para p<0.05. River Esera fish population density and biomass (with standard deviation). Comparison between before and after the withdrawal (* significative differences $p<0.05$ ).

\begin{tabular}{|c|c|c|c|c|}
\hline & & río Esera (ES-3) & densidad (ind/m2) & biomasa $(\mathrm{gr} / \mathrm{m} 2)$ \\
\hline & antes & después & antes & después \\
\hline Ch. toxostoma & $0.034(0.090)$ & $0.059(0.345)$ & $0.49(0.55)$ & $0.1480 .09)$ \\
\hline B. graellsii & $0.001(0.001)$ & $0.017(0.016)$ & $0.06(0.10)$ & $0.64(0.95)$ \\
\hline G. gobio & $0.025(0.014)$ & $0.080(0.149)$ & $0.06(0.05)$ & $0.11(0.12)$ \\
\hline TOTAL & $0.063(0.036)$ & $0.219(0.185)$ & $0.85(0.57)$ & $1.05(1.19)$ \\
\hline
\end{tabular}



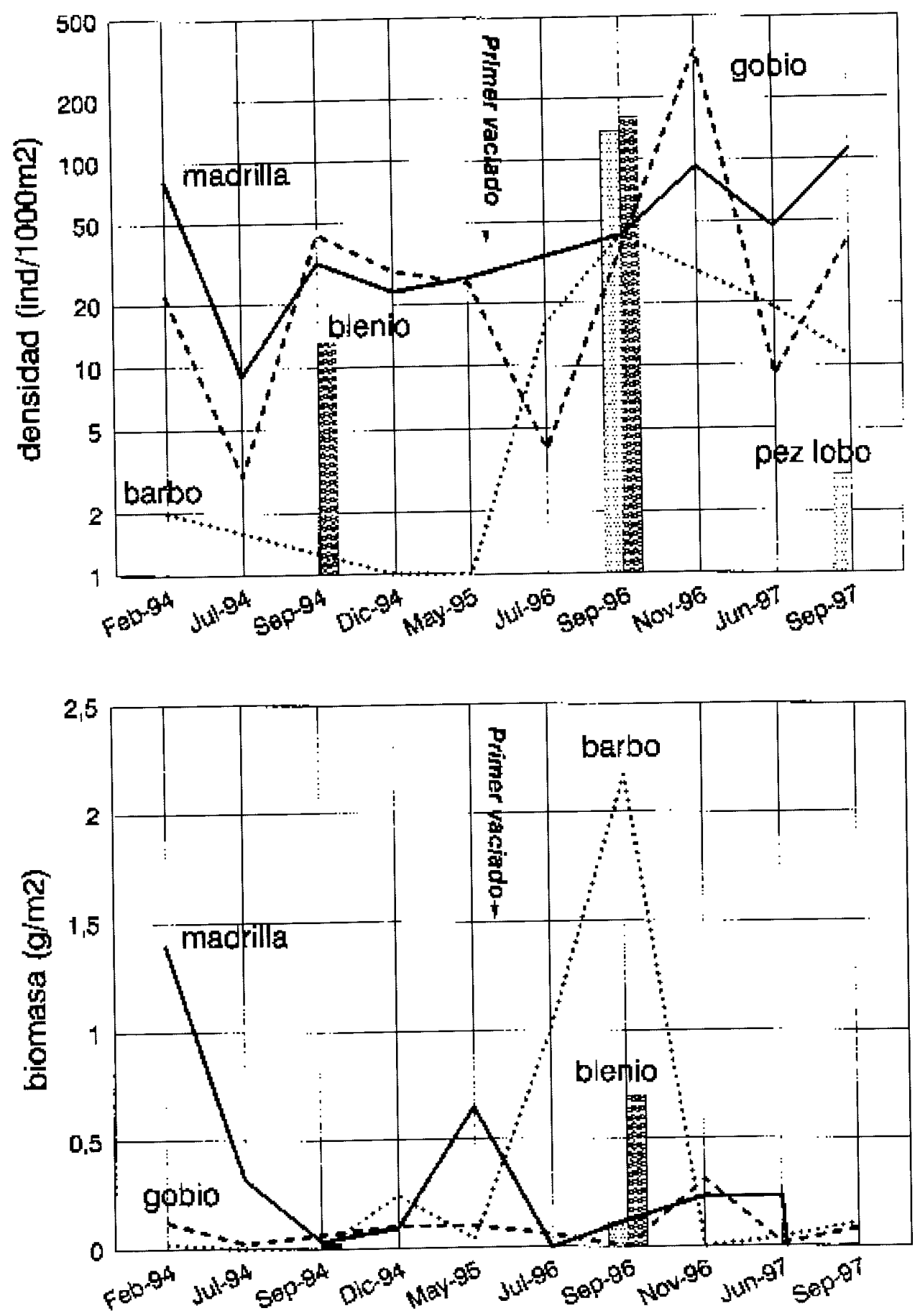

Figura 3. Evolución de la densidad y la biomasa de las distintas poblaciones ícticas del río Esera aguas abajo del embalse (ES-3) a lo largo del estudio. Density and biomass evolution of fish populations at Esera river (ES-3) during the study period. 
El barbo, cuya presencia en este tramo antes del vaciado era anecdótica, aumenta sus poblaciones después del mismo (Fig. 3). Además se observa una mejora (rejuvenecimiento) en su estructura poblacional: antes del desembalse la población estaba sumamente envejecida, con tres clases de edad $(2+, 3+$ y $7+)$. Tras el primer vaciado, en julio de 1996 aparecen algunos alevines y en septiembre dos tercios de la población son juveniles del año. En noviembre desaparece el barbo en este tramo, volviendo a aparecer en junio del año siguiente con una población compuesta en su mayoría por alevines (85\%). En septiembre la población está totalmente rejuvenecida, con alevines y juveniles en una proporción del $60 \%$ y $40 \%$ respectivamente.

La trucha común aparece de un modo esporádico tanto antes como después del desembalse, respondiendo a las repoblaciones que se hacen en el Cinca y los ocasionales casos en que se dan condiciones aptas para la trucha en el tramo.

Por otra parte, la carpa presenta unas poblaciones que aparecen ocasionalmente en este tramo (debido a escapes del embalse) adaptándose bien a las condiciones estivales para ser arrastrados en la crecidas posteriores. Después del desembalse se han vuelto a capturar varios alevines, procedentes probablemente de repoblaciones efectuadas en el embalse.

Las poblaciones de black-bass, al igual que las de carpa, dependían de los escapes ocasionales del embalse y desaparecen completamente después del vaciado.

Por último, dentro de las especies bentónicas, el blenio y el pez lobo tan solo se detectaron en septiembre de 1996, en el que resultaron ser las especies dominantes de la comunidad, debido quizás a condicionantes extremos del hábitat (fondos cubiertos de fango inertes y aguas con marcada turbidez) que perturbaba la presencia de otras especies. Es importante destacar la presencia del blenio de río, especie catalogada como En Peligro de Extinción (E) (ICONA, 1986). Está catalogada además como especie "de interés especial" en el Real Decreto 439/ 1990 (BOE 5-490).

\section{Río Cinca}

La composición de las comunidades del río Cinca se asemeja a las de los tramos bajos del Esera, encontrándose fundamentalmente madri- 1la, gobio y barbo, acompañados de trucha, pez lobo, blenio y bagre. Esta última especie, el bagre, desaparece después del desembalsado.

Sorprendentemente, los efectos del desembalse en el tramo del río Cinca en que se estudian cualitativamente las poblaciones (CI-2) son positivos en términos de densidad y biomasa. En efecto, la densidad media aumenta de $0.18 \mathrm{ind} / \mathrm{m}^{2}$ antes del desembalse a $0.44 \mathrm{ind} / \mathrm{m}^{2}$ después. Con la biomasa se observa el efecto opuesto, baja ligeramente (de $2.83 \mathrm{~g} / \mathrm{m}^{2}$ a $2.55 \mathrm{~g} / \mathrm{m}^{2}$ ).

Todavía más curioso es el hecho de que la variabilidad estacional de estas comunidades disminuye. Así, el coeficiente de variación (CV) en términos de densidad pasa del 55\% antes del desembalse al $47 \%$ después y, en términos de biomasa del $69 \%$ al $43 \%$ (Fig. 5).

En cuanto a la composición de la comunidad después del desembalse se mantiene la presencia de todos las especies que había antes con lo que la riqueza faunística para este tramo de río se mantiene constante ( 5 taxa) al igual que la diversidad que permanece en 0.53 bits, un valor bastante bajo. Sin embargo, las abundancias de las distintas poblaciones varían. Así, antes del desembalse la comunidad estaba dominada por la madrilla en invierno, por el gobio en primavera y en verano por el barbo; después del desembalse, la madrilla se convierte en la especie dominante a lo largo del año 96. No obstante, en 1997 parece sufrir una fuerte caída alcanzando en septiembre una densidad de $0.019 \mathrm{ind} / \mathrm{m}^{2}$ (carecemos de los datos correspondientes al mes de junio para corroborar o rechazar esta hipótesis).

La madrilla en el río Cincà se ve favorecida por el desembalse aumentando sus efectivos en biomasa y principalmente en densidad, al tiempo que estabiliza sus fluctuaciones (el CV de la biomasa pasa de $147 \%$ a $101 \%$ ). Después del vaciado, la densidad cuadruplica su valor (Tabla 3), mientras que su biomasa permanece prácticamente igual, dado que se han eliminado los individuos grandes (reproductores), los que más contribuyen a la biomasa total de la población. Si analizamos su estructura podemos observar, que antes del desembalse ésta es inestable, compuesta en su mayoría por adultos $(>1+)$, alcanzando sus cotas más elevadas en diciembre y febrero (Fig. 4). Ello parece indicar la existencia de migraciones de freza. Después del vaciado la población adquiere una estructura más estable, 

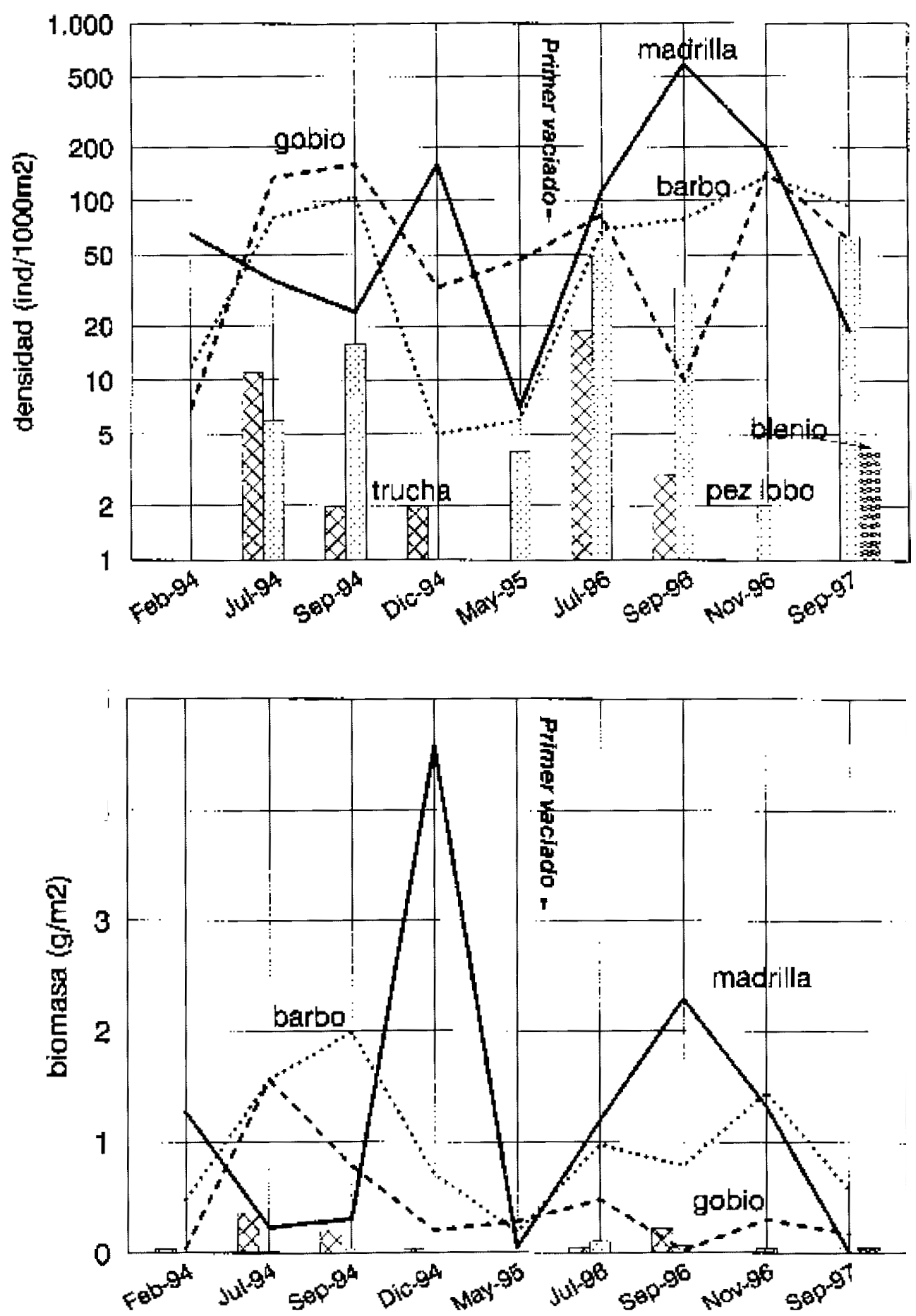

Figura 4. Evolución de la densidad y la biomasa de las distintas poblaciones íclicas del río Cinca (CJ-2) a lo largo del estudio. Density and biomass evolution of fish populations at Cinca river (CI-2) during the study period. 
Tabla 3. Densidad y biomasas medias (desviaciones estándar entre paréntesis) de las poblaciones ícticas del río Cinca antes y después del desembalse. " se observan diferencias significativas para $\mathrm{p}<0.05$. River Cinca fish population density and biomass (with standard deviation). Comparison between before and after thr withdrawal $(*$ significative differences $p<0.05)$.

\begin{tabular}{|c|c|c|c|c|}
\hline & & río Cinca (CI-2) & densidad (ind/m2) & biomasa $(\mathrm{gr} / \mathrm{m} 2)$ \\
\hline & antes & después & antes & después \\
\hline Ch. toxostoma & $0.058(0.061)$ & $0.231(0.253)$ & $1.29(1.90)$ & $0.96(0.97)$ \\
\hline B. graellsii & $0.042(0.047)$ & 0.097 (0.027)" & $0.98(0.76)$ & $0.78(0.53)$ \\
\hline G. gobio & $0.076(0.067)$ & $0.075(0.056)$ & $0.43(0.34)$ & $0.76(0.53)$ \\
\hline N. barbatulus & $0.005(0.006)$ & $0.037(0.026)^{*}$ & $0.01(0.02)$ & $0.10(0.10)$ \\
\hline TOTAL & $0.185(0.107)$ & $0.444(0.241) *$ & $2.83(1.86)$ & $2.56(1.09)$ \\
\hline
\end{tabular}

dominada por alevines del año y juveniles (clases $0+\mathrm{y} 1+$ ).

El barbo aparece en el Cinca con un número bastante bajo de individuos y con una estructura inestable repartida en siete clases de edad $(0+$ a $6+)$ dominada por los individuos de la clase $2+$. Tras el vaciado desaparece la clase $6+$ y la población se rejuvenece, adquiriendo una estructura mucho más estable, con un máximo de adultos en julio (periodo de freza) y un máximo de alevines en septiembre. En términos medios tanto la densidad como la biomasa de esta especie aumentan después del desembalse, al tiempo que disminuye su variabilidad estaciona1 (el CV pasa de $115 \%$ a un $28 \%$ ) lo que indica una estabilización de la población.

En cuanto a las poblaciones de gobio, antes del vaciado, se observan fluctuaciones de abundancias opuestas a la tendencia en el Esera, con un mínimo marcado en febrero (Figs. 3 y 4) aumentando más o menos progresivamente hasta septiembre, mes en que alcanza la máxima densidad registrada en todos los muestreos; disminuye en diciembre y se recupera a partir de mayo. Después del desembalsado, la densidad de la población de gobio sigue aumentando, aunque en septiembre sufre una fuerte caída. En términos medios la densidad del gobio se mantiene igual, mientras que la biomasa aumenta bastante $\left(\mathrm{B}_{\mathrm{m}}=0.43 \mathrm{~g} / \mathrm{m}^{2}\right.$ antes y $0.76 \mathrm{~g} / \mathrm{m}^{2}$ después del desembalse respectivamente). No obstante no existen diferencias significativas entre los dos periodos al $95 \%$ en ninguno de los parámetros poblacionales analizados. Por otra parte, su variabilidad temporal desciende ( $\mathrm{CV}=86 \%$ y $72 \%$ antes y después del vaciado). Al analizar su estructura poblacional, podemos observar que, antes del vaciado, ésta es inestable, con una práctica ausencia de alevines del año, al contrario de lo observado en el río Esera (las máximas densidades se alcanzan en los meses de septiembre y noviembre). Después del desembalse, la población se estabiliza aumentando el porcentaje de alevines y juveniles (clases $0+$ y $1+$ ).

Las capturas de pez lobo han sido, en general escasas pero las estimaciones de abundancia de sus poblaciones señala también un aumento significativo de las mismas después del desembalse (Tabla 3). Las poblaciones bentónicas, gobio y pez lobo, se ven favorecidas por el descenso de las aguas y su densidad y biomasa relativas aumentan, pasando a ser el grupo subdominante de la comunidad en el último muestreo (septiembre 97). El gobio requiere un sustrato arenoso o de gravas con macrófitas (no recubierto de fango), con una corriente moderada, mientras que el pez lobo prefiere aguas corrientes someras y claras, también con fondos de piedra, grava o arena (Doadrio et al, 1991). Estas son básicamente las condiciones reinantes durante el muestreo de septiembre.

\section{DISCUSIÓN}

El impacto de una actuación humana en las comunidades ícticas depende, por un lado, del tipo e intensidad de dicha actuación, y por otro, de la fragilidad y del estado de conservación de dichas comunidades. Lógicamente si el estado de las comunidades de peces es ya lo bastante degradado, las actuaciones que afectan a estas comunidades tendrán escaso impacto. Este hecho ha de 

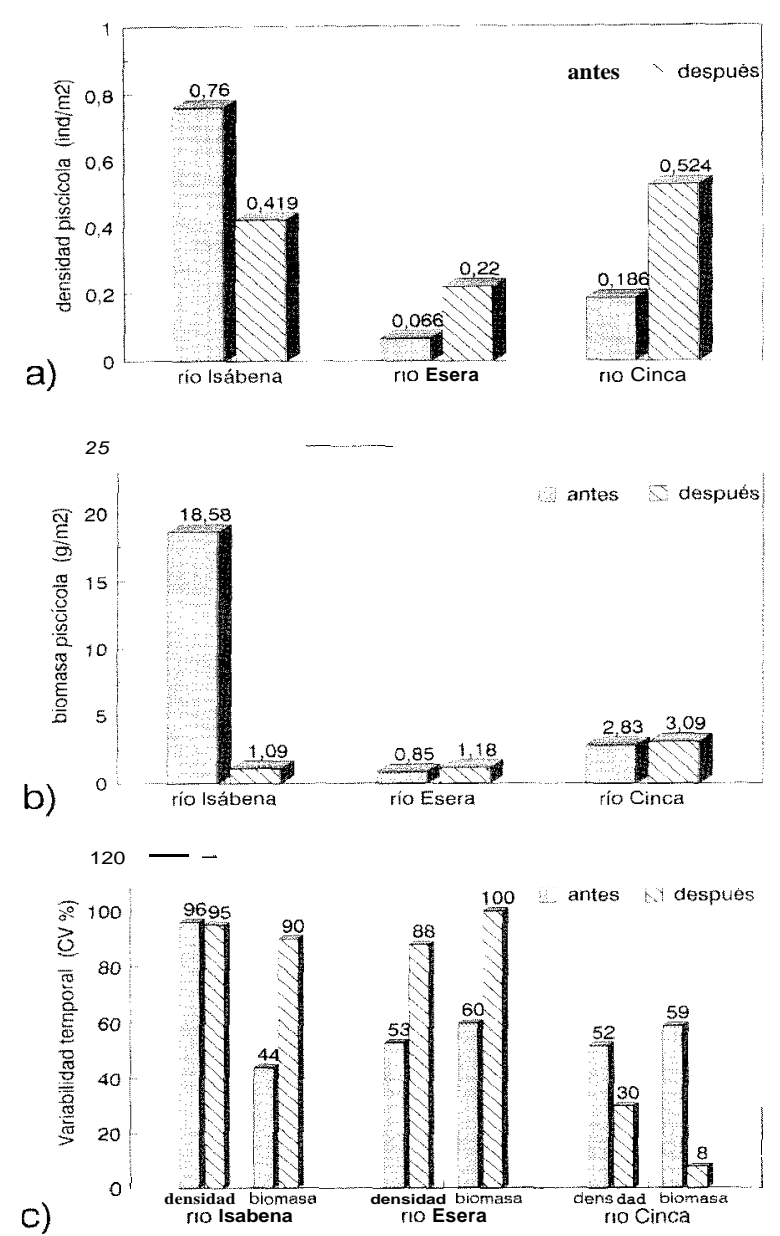

Figura 5. Efectos del desembalse en las comunidades ícticas fluviales: a) densidades medias: b) biomaaas medias; c) variabilidad temporal. Effects of the complete withdrawal over fluvial fish assemblages: $u$ ) average density; h) average biomass; $c$ ) variation coefficient.

ser tenido en cuenta a la hora de explicar los resultados de este trabajo.

Resulta sorprendente que los efectos del desembalse en las comunidades de peces, en síntesis, sean positivos en los tramos del Esera y del Cinca situados aguas abajo de la presa, mientras que son negativos en los tramos aguas arriba del embalse. En efecto, tanto la densidad como la biornasa medias aumentan después del desembalse en los tramos de aguas abajo, y, por el contrario, disminuyen en los de aguas arriba (Fig. 5).

La presencia del embalse de Barasona actúa como barrera asirnétrica que separa las comuni- dades ícticas de aguas arriba de la presa con las de aguas abajo. Estas últimas a su vez se ven más afectadas por la forma en que se gestiona el recurso hídrico.

Sería de esperar que la fuerza de arrastre de la corriente y los sedimentos que acarreara producidos por el desembalse, arrasara las poblaciones ícticas en los tramos de aguas abajo, mientras que los de los tramos aguas arriba no parece que debería ser afectadas. Sin embargo, nos hemos encontrado con que las comunidades de aguas abajo ya estaban muy deterioradas por otros impactos, antes del primer desembalse. El hecho de que el caudal del río sea extraído del cauce y desviado hacia el Canal de Aragón y Cataluña, restringe al caudal circulante por el cauce las filtraciones de la presa y a las ínfimas aportaciones de arroyos afluentes en el tramo restante de Esera. De esta forma el río se convierte en un rosario de pozas unidas por un hilillo de agua corriente. En este hábitat lenítico se ven favorecidas especies de aguas remansadas que de otra forma no vivirían en el río como la carpa y el black-bass. Por el contrario, en épocas de lluvias y de deshielos las crecidas arrasan con estas poblaciones leníticas y permiten la colonización por especies lóticas hasta que vuelve a desaparecer el caudal.

En los tramos de aguas arriba el río Esera tiene una comunidad muy simplificada por problemas con sedimentos en el fondos (provenientes de la erosión de ladera en la cuenca vertiente) y por la regulación hidroeléctrica que provoca bruscos cambios en los caudales circulantes. Por el contrario, el río Isábena tiene unas condiciones más naturales que le permiten sostener unas poblaciones ícticas importantes. Hemos visto que las poblaciones reófilas del embalse de barbo y madrilla utiliza casi exclusivamente los tramos de aguas arriba como zonas de desove y cría. Como consecuencia del desembalse la gran mayoría de los barbos del embalse fueron arrastrados aguas abajo o perecieron en el vaso, y por tanto en el río las poblaciones de peces disminuyeron en tamaño (Fig. 5a), en especial en biomasa, pues son los adultos los que desaparecen (Fig. 5b)

Elliott (1993) ha sugerido que la variabilidad temporal de las poblaciones de peces fluviales responde al tipo de factores que controla su mortalidad. Así, las poblaciones muy fluctuantes están controladas por factores físicos (crecidas, 
estiajes, sedimentación, etc.) y por tanto están mal estructuradas y son poco predecibles. En este sentido hemos evaluados la variabilidad temporal mediante el coeficiente de variación de la densidad y la biomasa poblacional de antes y después del desembalse.

En la figura $5 \mathrm{c}$ se exponen los efectos del desembalsado en la variabilidad temporal de las poblaciones ícticas. Vemos que en los tramos aguas abajo tenemos dos respuestas: en el río Ésera aumenta la variabilidad mientras que en el Cinca disminuye. Este resultado es coherente debido a que el río Cinca tiene una capacidad mucho mayor para amortiguar las avenidas producidas por el desembalse. En los tramos de aguas arriba (río Isábena) vemos como es la biomasa donde se produce un aumento significativo de la variabilidad, mientras la densidad no es afectada por el desembalse.

\section{CONCLUSIONES}

La conclusión más clara que se puede extraer a la vista de los resultados obtenidos es que el estado de las poblaciones ícticas en todos los tramos estudiados es bastante deficiente, tanto en sus aspectos cuantitativos (densidad y biomasa) como cualitativos (composición y estructura). Son poblaciones que sufren fuertes oscilaciones fruto del escaso caudal circulante, además de las migraciones reproductivas.

El río Isábena en principio parece ser el que ha sufrido un cambio más drástico en su comunidad íctica que se ha visto fuertemente reducida tanto en biomasa como en densidad. Esto parece perfectamente lógico dado que el embalse actuaba a modo de reservorio alojando a los individuos de mayor tamaño, reproductores potenciales.

En un principio el desembalsado ejercería un efecto de "barrido" de todo lo que hubiera aguas abajo (preferentemente individuos grandes que no pueden encontrar refugio ante la crecida de las aguas). Pero en el Esera, dado que las poblaciones eran escasas y estaban mal estructuradas (debido a la intensa regulación de caudales con una ausencia total de caudales ecológicos a lo largo del año) apenas se observan diferencias, tan solo un ligero incremento en el tamaño de las mismas.

En el río Cinca los efectos tampoco son muy patentes, no hay un efecto claro debido a que está más alejado de la presa, tiene un cauce más grande y sobre todo porque resulta afectado por la regulación de los embalses de El Grado y de Mediano. Aun así se han observado aumentos estadísticamente significativos en las poblaciones de madrilla y pez lobo.

Quizás en vez de evaluar los efectos del desembalsado habría que plantearse evaluar los efectos de la presa que son patentes, y planificar una gestión del embalse que mitigue dichos efectos, soltando caudales ecológicos que permitan un mantenimiento de las poblaciones normales. En especial se considera muy necesario fijar un régimen de caudales sobre todo teniendo en cuenta la presencia de especies protegidas como es el blenio de río.

\section{BIBLIOGRAFIA}

CARLE, F.L. \& M.R. STRUB. 1978. A new method for estimation population size from removal data. Biometrics, 34:621-680.

COWX, I.G. 1990. Developments in electric fishing. Fishing New Books. 358pp.

COWX, I.G., \& R.A. GOULD. 1985. The effects of short-term regulation releases from an impoundment on downstream fish fauna. Aquu. Fish. Manag., 1:257-264

GRANADO, C. 1981. Estudio ictiológico de los embalses de Cíjara, García de Sola y Zújar, durante su vaciado. Universidad de Sevilla.

ICONA. 1986. Lista roja de los vertebrados de España. Publicaciones del Ministerio de Agricultura. Madrid, 400 pp.

ELLIOT, J.M. 1993. The pattem of natural mortality throughout the life cycle in contrasting populations of brown trout, Salmo trutta L. Fish. Res., 17:123-136.

GARCÍA DE JALÓN, D., M. MAYO, F. HERVELLA, E. BARCELO y T. FERNÁNDEZ. 1993. Principios de gestión de la pesca en aguas continentales. Ed. Mundi-Prensa. Madrid.

LOBÓN-CERVIÁ, J. 1996. Response of a stream fish assemblage to a severe spate in Northern Spain. Trans. Am. Fish. Soc., 125 (6):913-920.

PRENDA J. y E. MELLADO. 1993. Características biológicas y espectro trófico durante el otoño de dos poblaciones simpátricas de Blenius fluivatilis y Micropterus salmoides en un embalse pequeño. Limnética, 9: 107-115 